\title{
NOMENCLATURA UNESCO: EVOLUCIÓN, ALCANCE Y REUTILIZACIÓN EN CLAVE ONTOLÓGICA PARA LA DESCRIPCIÓN DE PERFILES CIENTÍFICOS
}

\section{Juana-María Ruiz-Martínez, María-José Baños-Moreno y Rodrigo Martínez- \\ Béjar}

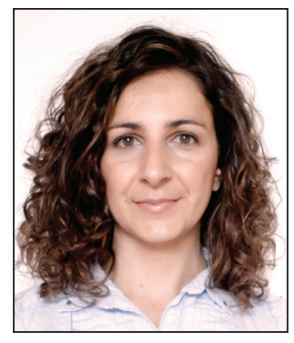

Juana-María Ruiz-Martínez es licenciada y doctora en documentación y licenciada en filología hispánica por la Universidad de Murcia. Sus líneas de investigación incluyen procesamiento de lenguaje natural, web semántica y en especial el enriquecimiento automático de ontologías. http://orcid.org/0000-0002-7928-8312

jmruymar@um.es

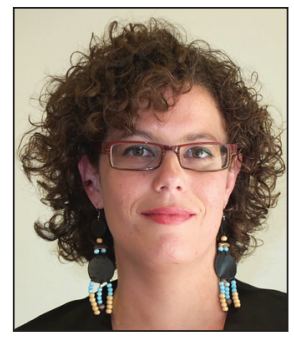

María-José Baños-Moreno es licenciada con premio extraordinario en documentación (2011) y máster en gestión de información (2013) por la Universidad de Murcia. Investiga en tecnologías de la web semántica en el Depto. de Ingeniería de la Información y las Comunicaciones de la Fac. de Informática de la misma universidad a través de una beca predoctoral.

http://orcid.org/0000-0001-9137-1330

mbm41963@um.es

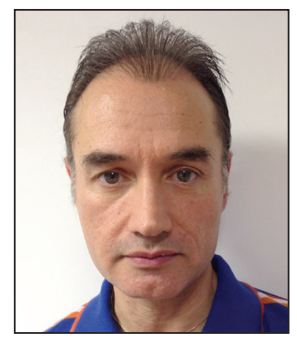

Rodrigo Martínez-Béjar es catedrático en la Facultad de Informática de la Universidad de Murcia y profesor visitante de la Univ. of New South Wales, Australia. Posee varias licenciaturas en las áreas de informática y sociología. Sus líneas de investigación incluyen la inteligencia artificial y la web semántica. Ha dirigido numerosos proyectos nacionales e internacionales y es coautor de más de 100 artículos publicados en revistas y conferencias.

http://orcid.org/0000-0002-9677-7396

rodrigo@um.es

Universidad de Murcia. Facultad de Informática Dpto. de Ingeniería de la Información y las Comunicaciones Campus de Espinardo. 30071 Murcia, España

\section{Resumen}

En 1974 la Unesco publicó la Nomenclatura Normalizada Internacional para los campos de Ciencia y Tecnología. Desde entonces se ha utilizado para la clasificación de la actividad científica en España. Con el objetivo de conocer mejor sus características, evolución y alcance se ha realizado un estudio bibliográfico y un análisis de su utilización en grupos de investigación universitarios y en otros ámbitos nacionales e internacionales. A partir de los datos obtenidos se ha diseñado una ontología basada en la combinación de la Nomenclatura y palabras clave. El resultado es un modelo ontológico adaptado a las tecnologías de la web semántica, que proporciona mayor expresividad que la Nomenclatura y soluciona problemas como la obsolescencia y la ausencia de ciertos temas, facilitando la clasificación de la actividad científica e incrementando su utilidad.

\section{Palabras clave}

Ontologías, Lenguajes documentales, Vocabularios, Nomenclaturas, Clasificaciones, Web semántica, Ciencia, Actividad científica, Nomenclatura Unesco de Ciencia y Tecnología.

Title: Unesco nomenclature: development, scope and reuse for describing scientist profiles using an ontology

\section{Abstract}

In 1974, Unesco published the Nomenclature for Fields of Science and Technology. Since then, it has been used in Spain to classify the scientific activity. In order to better determine its characteristics, development and scope, we studied the 
literature and analysed its use by university research groups in Spain and in other national and international settings. From the data obtained, we designed an ontology based on the combination of the Nomenclature and keywords identified in the literature. The result is an ontological model adapted to the semantic web technologies that provides a higher degree of expressiveness than the original Nomenclature. It also helps to solve problems such as obsolescence and the absence of certain emerging subject areas, facilitating the classification of scientific activities and increasing its usefulness.

\section{Keywords}

Ontologies, Documentary languages, Vocabularies, Nomenclatures, Classifications, Semantic web, Science, Scientific activity, Unesco Nomenclature for Fields of Science and Technology.

Ruiz-Martínez, Juana-María; Baños-Moreno, María-José; Martínez-Béjar, Rodrigo (2014). “Nomenclatura Unesco: evolución, alcance y reutilización en clave ontológica para la descripción de perfiles científicos". El profesional de la información, julio-agosto, v. 23, n. 4, pp. 383-392.

http://dx.doi.org/10.3145/epi.2014.jul.06

\section{Introducción}

La Nomenclatura para los campos de la ciencia y tecnología fue creada por la Unesco en 1973 y ha sido desde entonces una guía esencial para muchas organizaciones e instituciones internacionales, (Martínez-Frías; Hochberg, 2007). Implantada en España mediante Real Decreto (España, 1983a) es utilizada en las diversas administraciones del Estado para la clasificación de tesis doctorales, proyectos, grupos de investigación, investigadores, etc. Pese a su interés, uso y utilidad, desde 1988 no se ha actualizado. La ruptura con la realidad científica y tecnológica es evidente, puesto que las nuevas disciplinas y ramas surgidas en los últimos años no están contempladas en la clasificación.

\section{La ruptura de la Nomenclatura Unesco con} la realidad científica y tecnológica es evidente, las nuevas disciplinas surgidas en los últimos años no están contempladas

Por otro lado, los sistemas de gestión del conocimiento tradicionales no se adecuan al modo en el que la información se distribuye y comparte en la Red, por lo que su adaptación a este entorno es necesaria. Uno de los modelos utilizados en esta dirección son las ontologías, que en el contexto de la web semántica son capaces de dotar al conocimiento de un dominio de una estructura formal, reutilizable y compartible (Berners-Lee; Hendler; Lassila, 2001). Entre sus principales inconvenientes destaca el coste de actualización, por lo que es frecuente el uso de vocabularios ya consensuados como punto de partida.

La Nomenclatura de la Unesco, aún con sus carencias a nivel de obsolescencia, representatividad y usabilidad, es un sistema estandarizado y ambicioso que contiene buena parte de las áreas del conocimiento. Por ello constituye un buen punto de partida para la creación de un modelo ontológico que permita una mejor clasificación de la producción científica.

Los objetivos de este trabajo son:

1) estudiar el desarrollo y alcance de la Nomenclatura;

2) solventar el problema de su obsolescencia sin modificar la estructura original;
3) adaptarla a las necesidades y posibilidades de la web semántica.

La primera parte del artículo incide en las características, evolución e implantación de la Nomenclatura, así como en la experiencia de los usuarios con la misma. Se lleva a cabo una revisión bibliográfica, se muestran algunos casos de uso y se analiza su grado de implantación en la clasificación de la actividad de los grupos de investigación en universidades españolas. Se pone así en evidencia la problemática del ajuste a un sistema de categorización de la ciencia y la tecnología tomado como universal. En la segunda parte se propone un modelo ontológico basado en la combinación de palabras clave y códigos Unesco que pretende suplir las carencias de la Nomenclatura, devolviéndole su carácter normalizador. Finalmente se indican algunos resultados y conclusiones y se proponen mejoras del sistema diseñado.

\section{Nomenclatura de la Unesco: evolución y alcance}

En 1966 la Unesco se propuso desarrollar un sistema estándar internacional para la clasificación de la ciencia y la tecnología (Martínez-Frías; Hochberg, 2007, citando a Unesco, 1966). En 1973 su Oficina de Estadística y la División de Política Científica diseñaron el proyecto de Nomenclatura normalizada internacional para los campos de ciencia y tecnología. Su propósito era facilitar la cooperación internacional y el intercambio de información estadística científica, esto es, normalizar los datos estadísticos generados por las organizaciones científicas y tecnológicas (Unesco, 1974, p. 114). En 1974 fue publicada en inglés, francés y español con una vocación inicialmente restringida al ámbito administrativo.

La Nomenclatura fue adoptada en España en 1983 para la categorización de puestos de trabajo del CSIC mediante Real decreto 1804/1983 de 23 de mayo (España, 1983a). Ese mismo año se publicó la relación total de campos, disciplinas y subdisciplinas de especialización científica y tecnológica (España, 1983b), y comenzó a ser utilizada por buena parte de las instituciones científicas del país como forma de clasificación del personal de las áreas de ciencia y tecnología.

Tiene una estructura de tres niveles (cuatro en el caso español), de mayor a menor generalidad, conectados entre sí mediante relaciones jerárquicas y referencias cruzadas, como se presenta en la tabla 1. 
Tabla 1. Estructura de la Nomenclatura Unesco

\begin{tabular}{|c|l|c|l|l|}
\hline Nivel & \multicolumn{1}{|c|}{ Tipo } & Dígitos & \multicolumn{1}{|c|}{ Descripción } & Ejemplo \\
\hline $1^{\circ}$ & Códigos & 2 & Campos generales que clasifican las actividades científico-tecnológicas & 23. Química \\
\hline $2^{\circ}$ & Disciplinas & 4 & Describen detalladamente la actividad científica-tecnológica. & 2302. Bioquímica \\
\hline $3^{\circ}$ & Subdisciplinas & 6 & Profundiza en las actividades que forman parte de una disciplina & 2302.24. Péptidos \\
\hline $4^{\circ}$ & Especialidad & 8 & Sólo existen en la versión del CSIC & 2302.24-01. Síntesis de péptidos \\
\hline-- & Referencias & -- & Referencias cruzadas entre ítems & 2302. Bioquímica (Ver 2306) \\
\hline
\end{tabular}

La Unesco y el CSIC efectuaron actualizaciones que ampliaron la clasificación con nuevos códigos hasta 1988, fecha en que se incorporan los últimos datos (España, 1985a; 1985b; 1986; 1988; Unesco, 1988). En respuesta a la solicitud por parte de la International conferences on design history and studies de inclusión de la categoría "Diseño" (Icdhs, 1999; [2012]), ErdeIen (2002), asistente director general de ciencias naturales de la Unesco, confirmó que dicho organismo había abandonado el proyecto definitivamente en 1992. Esta evolución se recoge en la tabla 2 y con más detalle en la tabla 3.

La Nomenclatura tiene tres niveles (cuatro en el caso español) de mayor a menor generalidad, conectados entre sí mediante relaciones jerárquicas y referencias cruzadas

La Unesco y el CSIC desarrollaron la clasificación de forma divergente. Aunque ambas organizaciones tuvieron como punto de partida la publicación de 1974, la Unesco no incorporó las modificaciones posteriores del CSIC ni éste tuvo en cuenta los cambios introducidos por el organismo internacional a finales de 1988. Si la Nomenclatura aprobada por el CSIC en 1983 replicaba sin más la clasificación Unesco de 1974, las resoluciones de 1985, 1986 y 1988 modificaron el listado incorporando 4 disciplinas, 69 subdisciplinas y 32 especialidades (este último nivel no contemplado por el organismo internacional). La tabla 3 incide en esas diferencias.

Estas modificaciones, quizá poco conocidas, y la reutilización parcial del texto desde distintas entidades han provocado errores. La mayoría vienen por no incluir las disciplinas, subdisciplinas y especialidades añadidas por el CSIC.

Tabla 2. Evolución de la Nomenclatura Unesco de ciencia y tecnología

\begin{tabular}{|c|l|l|}
\hline Año & Entidad & \multicolumn{1}{c|}{ Acción } \\
\hline 1974 & Unesco & $\begin{array}{l}\text { Aprobación del Proyecto de nomenclatura } \\
\text { internacional normalizada en materia de } \\
\text { ciencia y de tecnología }\end{array}$ \\
\hline 1983 & CSIC & $\begin{array}{l}\text { Inclusión de la Nomenclatura en el sistema } \\
\text { científico para convocatoria de plazas en } \\
\text { CSIC; Publicación de la relación total de } \\
\text { campos, disciplinas y subdisciplinas }\end{array}$ \\
\hline 1985 & CSIC & Actualizaciones de la Nomenclatura \\
\hline 1986 & CSIC & Actualización de la Nomenclatura \\
\hline 1988 & $\begin{array}{l}\text { CSIC } \\
\text { UnesCo }\end{array}$ & $\begin{array}{l}\text { Actualización } \\
\text { Actualización }\end{array}$ \\
\hline 1992 & Unesco & Abandono del programa de Nomenclatura \\
\hline
\end{tabular}

Por ejemplo, las réplicas de la Universidad de Lleida y del País Vasco recogen sólo las modificaciones de 1985 y 1986, y la versión SKOS (Pastor-Sánchez, 2012) no incluye los códigos definidos por el CSIC.

http://www.udl.cat/recerca/RDIhtml/unesco.html http://www.et.bs.ehu.es/varios/unesco.htm

\section{La nomenclatura en la ciencia: usos y aplicación}

Este estudio comprende una revisión bibliográfica, un análisis específico de los diferentes sistemas de clasificación de dicha actividad en las webs de grupos de investigación de universidades españolas y una muestra de casos de uso más allá de estos grupos.

\subsection{Revisión bibliográfica}

Se ha pretendido conocer la relevancia de la Nomenclatura Unesco a nivel de publicación científica. Urdín-Camino y Morillo (2000) analizan la producción científica andaluza a partir de los datos del Science Citation Index (SCI) y el Índice Español de Ciencia y Tecnología (ICYT), clasificando los artículos de cada base de datos por los campos científicos Unesco. Los campos más prolíficos fueron ciencias de la vida, química (en $S C l$ ) y ciencias tecnológicas (en ICYT), entre otros. UrdínCamino (2001) clasifica las revistas españolas recopiladas en los directorios del Cindoc a partir de la Nomenclatura. Como contribución a la guía de buenos usos para la edición de revistas científicas, ofrece una panorámica general de las publicaciones periódicas científicas del país. Urdín-Camino y Alcaín (2004) describen las bases de datos del CSIC, entre cuyas características se encuentra la clasificación de artículos por la Nomenclatura, siendo posible su posterior recuperación mediante un campo ad hoc en la ventana de búsqueda.

Por su parte, Fernández y Peral (2007), en un trabajo de carácter histórico, estudian 132 números del Boletín Oficial de la Provincia de Badajoz de 1880 y catalogan sus 1.027 noticias empleando la Nomenclatura Unesco, que consideran "de carácter internacional, suficientemente referenciada y muy conocida por la comunidad científica".

Tabla 3. Evolución de la Nomenclatura por año, institución y campos

\begin{tabular}{|c|l|r|r|r|r|r|r|}
\hline Nivel & \multicolumn{1}{|c|}{ Tipo } & $\begin{array}{c}\text { Unesco } \\
\mathbf{1 9 7 9}\end{array}$ & $\begin{array}{c}\text { CSIC } \\
\mathbf{1 9 8 3}\end{array}$ & $\begin{array}{r}\text { CSIC } \\
\mathbf{1 9 8 5}\end{array}$ & $\begin{array}{r}\text { CSIC } \\
\mathbf{1 9 8 6}\end{array}$ & $\begin{array}{c}\text { CSIC } \\
\mathbf{1 9 8 8}\end{array}$ & $\begin{array}{c}\text { Unesco } \\
\mathbf{1 9 8 8}\end{array}$ \\
\hline $1^{\circ}$ & Códigos & 24 & 24 & 24 & 24 & 24 & 24 \\
\hline $2^{\circ}$ & Disciplinas & 245 & 245 & 248 & 249 & 249 & 245 \\
\hline $3^{\circ}$ & Subdisciplinas & 2.180 & 2.180 & 2.210 & 2.237 & 2.249 & 2.183 \\
\hline $4^{\circ}$ & Especialidad & 0 & 0 & 6 & 17 & 32 & 0 \\
\hline-- & Referencias & 755 & 755 & 755 & 755 & 755 & 743 \\
\hline
\end{tabular}




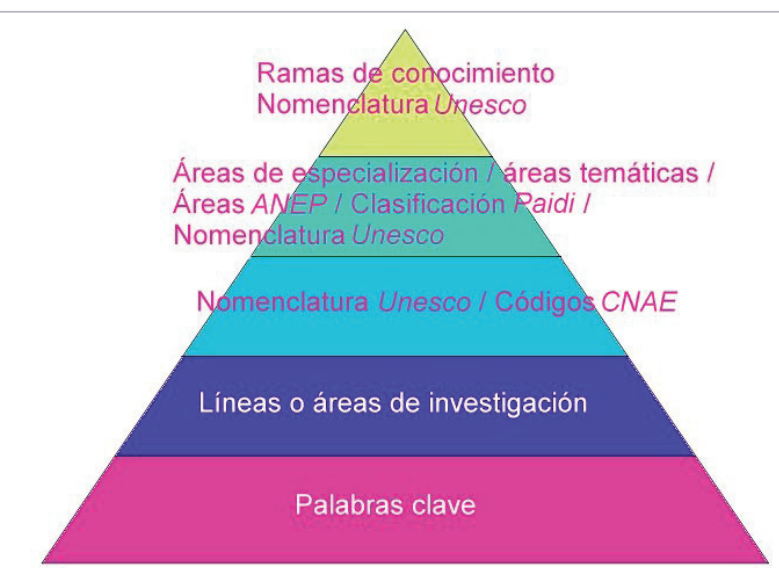

Figura 1. Herramientas de clasificación de la actividad investigadora en universidades españolas

Martínez-Frías y Hochber (2007) publican el único trabajo en el que el objeto de estudio es la propia Nomenclatura y ponen de manifiesto dos problemas:

- asignación errónea de determinadas disciplinas y subdisciplinas;

- necesidad de actualizar las categorías a medida que surgen y se asientan otras.

Ortega y Aguillo (2007) utilizan la clasificación para asignar categorías a las webs de departamentos y grupos de investigación de universidades españolas y así analizar el grado de interdisciplinariedad entre ellos. Los autores destacan como una de las principales dificultades del trabajo la jerarquía temática preestablecida de la clasificación, ya que podría "afectar a la detección de relaciones fronterizas entre disciplinas". Finalmente, Suárez y Baquero (2013) examinan los proyectos del sector agropecuario financiados por el Departamento Administrativo de Ciencia, Tecnología e Innovación (Colciencias, Colombia) en seis convocatorias y durante 2010. Su organización por disciplinas de la Nomenclatura es una más de las perspectivas (en este caso temática) desde la que se analizan dichos proyectos.

Los grupos de investigación emplean diferentes herramientas de clasificación y muchas veces de forma simultánea

\subsection{Sistemas de clasificación en las webs de grupos de investigación}

Se ha realizado un estudio de las herramientas de clasificación de la actividad científica utilizadas por los grupos de investigación de las cien universidades españolas más destacadas. La selección de universidades se ha realizado en función de la posición que ocupan en el Ranking Web de Universidades (CSIC, Laboratorio de Cibermetría, 2013). http://www.webometrics.info/es

A partir de la página principal de cada institución, mediante navegación o a través del buscador interno de la web, se ha accedido a las páginas de tres grupos de investigación seleccionados aleatoriamente. Las variables que se han ana- lizado son el uso de la Nomenclatura Unesco, el uso de otros métodos clasificatorios de la actividad científica, y la denominación de los métodos.

Los datos reflejan que los grupos de investigación emplean diferentes herramientas de clasificación y muchas veces de forma simultánea. Por ejemplo, la Universidad Complutense de Madrid codifica la actividad investigadora mediante Nomenclatura Unesco, palabras clave y líneas de investigación. Según su nivel de desarrollo, éstas pueden ser organizadas de mayor a menor grado de especificidad, tal como se muestra en la figura 1. La Nomenclatura, por su estructura y profundidad, se situaría en varios niveles de la clasificación.

Además de los métodos anteriores, se han hallado otras herramientas como las Áreas ANEP, la clasificación Paidi o la CNAE2009, representadas en la figura 1 en el mismo nivel que las áreas de especialización y la Nomenclatura Unesco, con las que podrían ser asimiladas, de acuerdo a su nivel de profundidad.

La Agencia Nacional de Evaluación y Prospectiva diseñó las denominadas Áreas ANEP para reflejar la situación actual de las actividades de I+D que se llevan a cabo en los centros de investigación y universidades españolas. Son utilizadas por ejemplo por las universidades de les Illes Balears y da Coruña.

http://goo.gl/hSfDde

La clasificación Paidi (Ponencias del plan andaluz de investigación, desarrollo e innovación $)^{1}$ fue creada por la Consejería de Innovación, Ciencia y Empresa andaluza y es explotada en las Universidades de Granada, Almería, Málaga y Pablo de Olavide.

La CNAE-2009² (Clasificación nacional de actividades económicas) fue aprobada mediante Real decreto 475/2007 como adaptación a la Nomenclatura estadística de actividades económicas de la Comunidad Europea (NACE) y tras el proceso internacional de revisión Operación 2007 para facilitar la clasificación de unidades estadísticas y entidades según la actividad económica ejercida. Es utilizada en las universidades de Alicante y Jaume I, aunque esta codificación se refiere a actividades empresariales y no siempre es válida para las actividades científicas.

http://www.ine.es/jaxi/menu.do?type=pcaxis\&path=\%2Ft4 $0 \% 2$ Fclasrev\%2F\&file=inebase $\& L=0$

Otros sistemas hallados son la clasificación por ámbitos temáticos de las universidades de Barcelona y Rovira $i$ Virgili; el nivel de consolidación de los grupos utilizado por la Universitat de Lleida; los códigos SIC en la Universidad de Sevilla; o clasificaciones especializadas como la Cerif (Common European research classification scheme), usada por el Grupo de Investigación de la Literatura y sus Aplicaciones de la Universidad de Granada.

http://www.arrs.gov.si/en/gradivo/sifranti/sif-cerif-cercs.asp

El gráfico 1 muestra las herramientas existentes para clasificar la actividad de los grupos de investigación y su nivel de utilización. Los métodos citados anteriormente, no indicados explícitamente en el gráfico, han sido englobados bajo la denominación "Otros".

Este estudio refleja la variabilidad de sistemas clasificatorios existentes en España. El más utilizado (67 universidades) 
son las líneas de investigación, seguido por las palabras clave (17), Nomenclatura Unesco (14), ramas de conocimiento (13) y áreas de especialización (9). Desde nuestro punto de vista la Nomenclatura es el último nivel normalizador de clasificación. Tanto palabras clave como líneas de investigación (los escalones con mayor grado de descripción), son subjetivos y quedan limitados al arbitrio de cada entidad, por lo que su estandarización es difícil.

\subsection{Usos de la Nomenclatura en la actividad científica}

La Nomenclatura también es utilizada para la clasificación de la actividad científica en otras situaciones diferentes a las ya presentadas. A continuación se muestran prácticas a nivel nacional (España) e internacional (Latinoamérica).

Para incluir cualquier tesis en la base de datos Teseo es requisito clasificarla según la Nomenclatura. Ello permite su recuperación posterior mediante un campo de palabras clave (códigos Unesco) en la ventana de búsqueda.

https://www.educacion.gob.es/teseo/irBusquedaAvanzada.do

También es muy habitual que en solicitudes de becas y proyectos de investigación, se deba completar algún campo en el que clasificar temáticamente nuestra investigación. Por ejemplo es requisito en los currículos para participar en el Subprograma Ramón y Cajal (convocatoria 2011).

Algunas universidades incluyen entre los requerimientos de tramitación de tesis doctorales su clasificación mediante categorías Unesco, por ejemplo en la Universidad de Navarra (Torregrosa, 2013). También en Honduras la Nomenclatura es utilizada para la ordenación de proyectos de investigación y tesis doctorales en el Sirih (Sistema de información y registro de los investigadores de Honduras).

http://www. sirih.org/?cat=1026\&title=NomenclaturaUnesco\&lang=es

Colón, el buscador de estudios de posgrado en España desarrollado por Universidad de Valladolid, Aedcid y el Ministerio de Asuntos Exteriores y Cooperación, recoge un campo de búsqueda por código Unesco.

http://www.buscadorcolon.org/posgrado.php

La Biblioteca de recursos Universia recoge millones de registros de artículos científicos publicados a nivel internacional, organizados y localizables en la base de datos por categorías de la Clasificación.

http://biblioteca.universia.net

Redes de colaboración es un portal creado por la Universidad de Valladolid para la localización de redes de trabajo académicas y también se organiza por códigos Unesco.

http://www.redesdecolaboracion.org/Index. php?MOD=Buscador $\& O P=10$

En Argentina el Caicyt (Centro Argentino de Información Científica y Tecnológica) creó un banco semántico como infraestructura de apoyo conceptual y terminológico para procesos de representación, búsqueda, descubrimiento e intercambio del conocimiento científico y tecnológico. Entre los vocabularios utilizados para estos procesos se encuentra la Nomenclatura.

http://vocabularios.caicyt.gov.ar/portal/home.php

En Ecuador, para la presentación de programas y proyectos

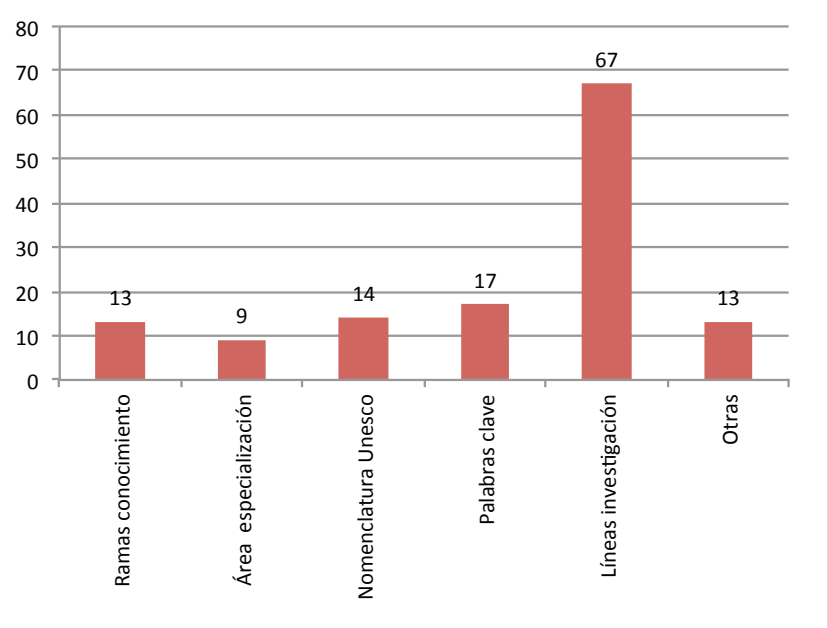

Gráfico 1. Herramientas de clasificación de la actividad de grupos de investigación en universidades españolas ${ }^{3}$

de investigación de la Universidad de Guayaquil/4 (convocatoria 2014), hay que especificar el área científica del trabajo de acuerdo con la Nomenclatura Unesco.

Finalmente, México cuenta con su propia versión de la Nomenclatura, publicada por el Consejo Quintanarroense de Ciencia y Tecnología (Consejo, 2000). Es conocida como Clasificación Barros.

\subsection{Problemas de la Nomenclatura en sus términos actuales}

La literatura científica analizada en el apartado 3.1 es en general poco relevante para el caso que nos ocupa. En ella, la Nomenclatura Unesco es utilizada como herramienta de clasificación de la actividad científica desde diferentes puntos de vista y sólo un artículo (Martínez-Frías; Hochber, 2007) la presenta como objeto de estudio. Si bien dos trabajos inciden en algunos inconvenientes de la Nomenclatura (Martínez-Frías; Hochber, 2007; Ortega; Aguillo, 2007), no existe un estudio exhaustivo que evalúe su empleo, utilidad y posibilidades. El subapartado 3.2 pone de relieve la presencia de varios sistemas de clasificación de la actividad llevada a cabo por los grupos de investigación. La Nomenclatura, pese a no ser la más utilizada, es conocida y normalizada. Los ejemplos indicados en el subapartado 3.3 muestran como, aún con las dificultades manifiestas, también es aplicada en otros ámbitos científicos, principalmente en España.

Los principales problemas de la Nomenclatura Unesco, una vez analizada la literatura y su uso en diferentes ámbitos, pueden resumirse en:

- Obsolescencia. La Unesco "debe estar abierta a la incorporación de nuevas y emergentes áreas de desarrollo científico" (Martínez-Frías; Hochber, 2007). Desde 1988 se han producido múltiples avances que han dado lugar a nuevas áreas científico-tecnológicas, actualmente no representadas en la clasificación. Por ejemplo, "Diseño" (IDCHS, 1999), "Enfermería” (Pedraz-Marcos, 2005) ó “Astrobiología" (Martínez-Frías; Hochber, 2007). Tampoco las tecnologías relacionadas con la Web están representadas. En estos casos es frecuente recurrir a "Otros (especificar)", aunque ello no aporta mayor información.

- Aunque existen referencias cruzadas debido a la interdis- 
ciplinariedad, es evidente que en su estado actual resultan insuficientes. Para Martínez-Frías y Hochber (2007) la subdisciplina "Geología planetaria" debería depender de "Ciencias del espacio y la tierra" y no sólo de "Astronomía y astrofísica". Por su parte, Ortega y Aguillo (2007) fusionan para su estudio "Ciencia de los ordenadores" y "Tecnología de los ordenadores" en "Informática" dejando entrever la necesidad de redefinir las relaciones existentes e incluso los propios códigos.

- Excesiva compartimentación. La multidisciplinariedad / interdisciplinariedad ha ganado terreno en la ciencia (Ortega; Aguillo, 2007) y es necesario recurrir a varios códigos, cuya suma no indica la idea de lo que se está clasificando. Por ejemplo, "Bioinformática".

- Errores en la asignación de disciplinas y subdisciplinas que no se han resuelto y se repiten sistemáticamente en las clasificaciones que se encuentran en la Red. Es el caso de las referencias incorrectas entre códigos. Un error común en las versiones en español es la redirección de "1203. Ciencia de los ordenadores" a "3309. Tecnología de los alimentos" en lugar de a "3304. Tecnología de los ordenadores".

- Compartición de espacio con otras herramientas clasificatorias, que puede dificultar su estandarización y expansión, ya que las organizaciones han dedicado esfuerzos a adaptar o crear otros sistemas.

\section{El modelo ontológico diseñado tiene como base la Nomenclatura y se com- plementa con palabras clave}

\section{Propuesta de modelo ontológico basado en la Nomenclatura}

La codificación de la Unesco, pese a los problemas señalados, no deja de ser un modelo general, estándar y reutilizable, que recoge buena parte de las ramas del conocimiento, bajo las cuales se podrían agrupar las más específicas. El empleo de diferentes sistemas de clasificación no hace más que evidenciar la falta de sistematización a la hora de organizar la actividad científica y la necesidad de recuperar un sistema normalizado, como fue la Nomenclatura. Debido a su obsolescencia, la solución adoptada por parte de la comunidad científica es el recurso a las palabras clave y líneas de investigación, cuyo nivel de expresividad es más elevado, aunque difícilmente extensible a toda comunidad por su subjetividad y alto grado de especialización.

Se ha diseñado un modelo ontológico para establecer un nexo de unión entre palabras clave, líneas de investigación y códigos Unesco, y para proporcionar un soporte clasificatorio sobre el que realizar inferencias lógicas. El modelo tiene como base la Nomenclatura y se complementa con palabras clave o keywords. De esta forma se consigue mayor expresividad sin renunciar a una estructura jerárquica.

Se ha utilizado el lenguaje ontológico OWL2 (W3C, 2012) y se ha adoptado la metodología Methontology (FernándezLópez; Gómez-Pérez; Juristo, 1997). El punto de partida han sido los códigos de la Nomenclatura ${ }^{5}$, que se agrupan bajo la clase "Área de conocimiento". Cada código se ha definido como una clase y puede contener otras subclases de acuerdo a la estructura jerárquica de la Nomenclatura (códigos, disciplinas, subdisciplinas y especialidades). Los códigos se han definido como un metamodelo en el que cada clase contiene un individuo con el mismo nombre (punning).

Las descripciones lingüísticas se han incluido como etiquetas o labels y las referencias cruzadas se representan mediante la etiqueta see also. No se ha empleado la versión SKOS de la Nomenclatura, ya que a pesar de utilizar el estándar para transformar y representar en RDF los tradicionales sistemas de organización documental (Pastor-Sánchez, 2012), trata la codificación de un modo diferente a como se hace en este trabajo en el que se ha optado por el metamodelo, además de no contemplar las últimas modificaciones efectuadas por el CSIC. Se han excluido de la clasificación las categorías finalizadas en .99 con la etiqueta "Otras (especificar)" ya que no aportan conocimiento a la ontología.

En la figura 2 se muestra un extracto de la clase "Área de conocimiento" en el editor de ontologías Protègè .

En torno a esta clase se han creado otros conceptos, propiedades y axiomas, constituyéndose un modelo ontológico que permite describir la experiencia docente e investigadora combinando códigos Unesco y palabras clave. En la figura 3 se presenta un diagrama de los principales componentes de la ontología.

En esta representación los conceptos sombreados indican el núcleo ontológico o core ontology, en donde se organiza la experiencia de un usuario en función de las áreas de conocimiento y la experiencia profesional definida por el mismo. Este núcleo es exportable y expandible según las necesidades del dominio.

El concepto "Usuario" hace referencia a cualquier entidad del tipo persona que tenga al menos una relación con una "Organización" (universidad, empresa, centro de investigación, etc.) y una "Titulación académica". Un usuario puede pertenecer a dos categorías no excluyentes entre sí: docente e investigador. Un usuario-docente posee alguna experiencia docente definida mientras que un usuario-investigador posee alguna experiencia investigadora definida. La "Experiencia profesional" es la unión, de la experiencia docente y la experiencia investigadora.

Figura 2. Extracto de la ontología Nomenclatura Unesco, clase "Área de conocimiento" 
En la figura 3 se puede observar la clase "Investigador" y los axiomas que la definen.

Las palabras clave se introducen en la ontología como data properties y, como se ha dicho, son atributos de las instancias de la clase "Experiencia".

En el apartado 3.2. se mostró que los expertos relacionan un conjunto de palabras clave con un conjunto de códigos Unesco, de modo que una palabra clave puede estar relacionada con uno o más códigos. Es decir, es el conjunto de palabras clave y códigos lo que indica la experiencia global, y no la relación individual de una keyword con un código. En consecuencia cada instancia de la clase "Experiencia profesional" es el conjunto de "Áreas de conocimiento" y keywords indicadas por un usuario. A través de las relaciones tiene_experiencia_profesional_usuario y tiene_area_ de_conocimiento_profesional se relaciona una instancia de usuario con las "Áreas de conocimiento" pertinentes.

En este trabajo se descartó la posibilidad de añadir nuevas subclases a la Nomenclatura, así como relacionar directamente las palabras clave indicadas por un usuario con un código concreto, es decir, en una relación de uno a uno.

Además de las clases principales que se han descrito, el modelo ontológico cuenta con la clase "Área temática" que se ha instanciado con conjuntos de palabras clave y códigos Unesco extraídos aleatoriamente de las webs de los grupos de investigación analizados en el apartado 3.2. Su función es doble:

- contiene conocimiento inicial que permite realizar experimentos y facilita la obtención de resultados cuando el sistema se implementa por primera vez;

- permite incluir relaciones entre códigos de la Unesco y palabras clave sin estar relacionados con un usuario concreto.

Por su parte, la clase "Titulación académica" contiene un listado con todas las titulaciones académicas de educación superior?.

La tabla 4 muestra un resumen de las métricas de la ontología teniendo en cuenta todos los individuos y relaciones, tanto de las clases principales como de la clase "Área temática".

De las 2.357 clases, un total de 2.341 corresponden a la jerarquía de la clase "Área de conocimiento". En las object properties se han incluido las relaciones inversas y otras relaciones secundarias y subrelaciones. Las data properties son dos, las keywords que se han descrito y el CIF utilizado para identificar organizaciones. Y por último en los individuos, 9.713 son instancias de la clase "Titulación académica", 190 de "Área temática" y 2.341 de "Área de conocimiento".

Tabla 4. Métricas de la ontología diseñada

\begin{tabular}{|l|r|}
\hline Clases & 2.357 \\
\hline Object properties & 18 \\
\hline Data properties & 2 \\
\hline Individuos & 12.244 \\
\hline
\end{tabular}

\subsection{Descripción de un caso de uso de la ontología di- señada}

Se describe cómo integrar este modelo ontológico en una plataforma para la gestión de la experiencia (profesional) científica de los miembros de un grupo de investigación cualquiera. Uno de los principales retos en este tipo de proyectos es el uso de una ontología funcional, con las suficientes clases definidas para poder realizar inferencias, pero sin resultar demasiado restrictiva. De este modo se facilita su adaptación a distintos dominios y su utilización en diferentes aplicaciones. Los componentes básicos para la implementación del producto se pueden resumir en:

- Plataforma para el registro de usuarios desde el front-end, que permita al usuario introducir y validar códigos Unesco y palabras clave.

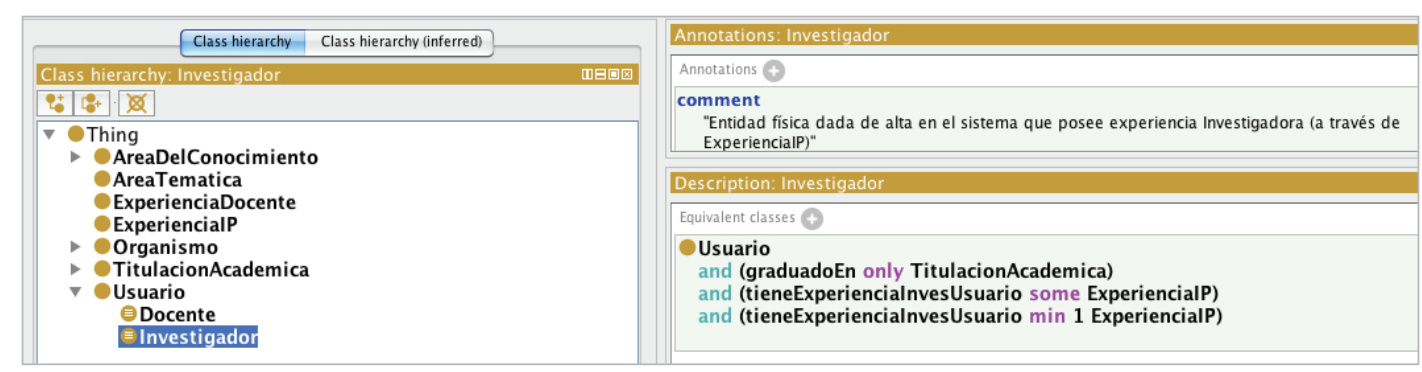

Figura 4. Extracto de la ontología diseñada, clase "investigador" 
- Gestión de palabras clave desde el back-end. La introducción de términos libres en el sistema supondría un problema para la estabilidad de la ontología. Por ello, se prevé un módulo desde el que el administrador puede gestionarlas. De este modo se puede controlar su entrada en la ontología y así evitar repeticiones o erratas. También es posible definir un procedimiento de conteo por el cual si una keyword es introducida cierto número de veces, se integra automáticamente en el sistema.

- Métodos para la instanciación y consulta de la ontología. Esta parte requiere el desarrollo de componentes software que permitan ejecutar los distintos casos de uso que se pretendan resolver con la ontología. Por ejemplo, insertar un nuevo usuario o modificar uno existente; consultar cuál es la experiencia investigadora de un usuario o buscar palabras clave ya existentes en la ontología.

- Consulta de la ontología desde el front-end. Permite realizar consultas dependiendo de los casos de uso desarrollados. Por ejemplo se puede saber qué usuarios tienen experiencia docente en determinadas áreas del conocimiento, pudiendo hacer una búsqueda más o menos restrictiva en función de los niveles jerárquicos de la Nomenclatura Unesco.

En la figura 5 se ilustra el funcionamiento del sistema.

En primer lugar el usuario indica las palabras clave relacionadas con su experiencia docente y/o investigadora. Con esta información el sistema realiza una búsqueda entre las labels de la ontología y las palabras clave introducidas por otros expertos y arroja un conjunto de códigos Unesco. Estos códigos son propuestos al usuario, que puede validarlos, descartarlos o añadir nuevos. El resultado es una instancia de la clase "Experiencia profesional" (a través de alguna de sus subclases) compuesta por las keywords que el usuario indicó y las áreas asociadas a las mismas.

Por ejemplo, una instancia de la clase "Área temática" (que contiene el conocimiento inicial para poder operar con la ontología) tiene asociadas las palabras clave "Acuicultura", "Biodiversidad", "Sistemas fluviales", "Impacto ambiental" y los códigos "2508.05. Hidrología”, "2508.08. Limnología”, "2508.14. Aguas superficiales", "2417.13. Ecología vegetal" y “3308.06. Regeneración del agua".

Si un usuario indica las palabras clave "Sistemas fluvia-

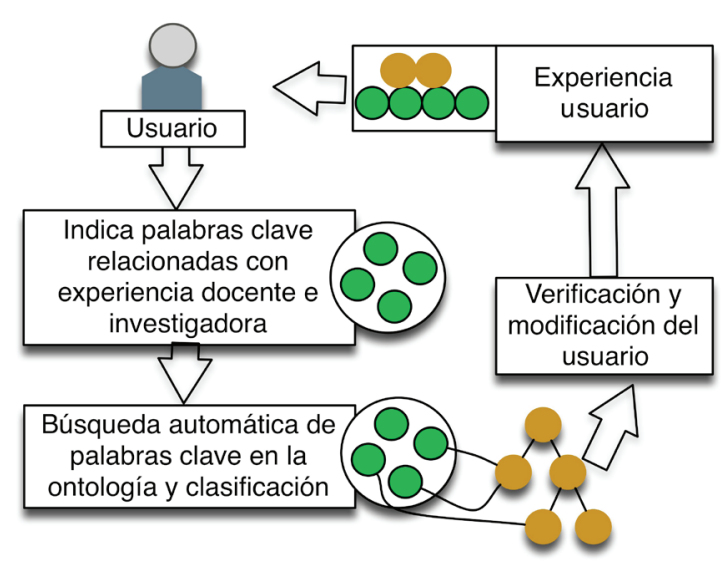

Figura 5. Esquema de funcionamiento del sistema les", "Cambio global", "Servicios ecosistémicos" y "Funcionamiento ecosistema", el sistema propone los códigos $2508.05,2508.08,2508.14,2417.13$ y 3308.06.

Después de analizarlo, el usuario opta por el código general 2508, selecciona el 3308.06 y descarta el 2417.13, constituyéndose así una nueva "Experiencia investigadora", esta vez asociada a un usuario concreto.

Las ventajas que este sistema comporta se pueden resumir en tres aspectos:

- el uso de palabras clave suple la falta de especificidad de la Nomenclatura y, en los casos de las áreas científico/tecnológicas con escasa representación, son imprescindibles para definir el ámbito de estudio. De hecho, en los grupos de investigación analizados en el apartado 3.2, la mayoría de los grupos se esfuerza en la elección de palabras clave, mientras que optan por códigos generales (normalmente de 4 cifras);

- los usuarios utilizan un amplio abanico de keywords, términos libres afectados por los problemas derivados del uso de lenguaje natural (polisemia, sinonimia o neologismos). Esto genera dispersión de la información e imposibilita identificar áreas y especialidades. El uso de la ontología contextualiza las palabras clave y posibilita su clasificación, ya que reduce la incertidumbre derivada de la falta de control;

- el modelo ontológico aprovecha la normalización de la Nomenclatura y la expresividad de las palabras clave. De este modo el grado de usabilidad aumenta ya que el experto no necesita revisar todos los códigos de la Nomenclatura para encontrar los más adecuados, puesto que es el sistema el que los sugiere en base a los recursos almacenados en la ontología.

\section{Conclusiones}

La Nomenclatura Unesco es un valioso punto de partida para la clasificación de la ciencia y la tecnología. La revisión bibliográfica y el análisis de casos de uso revelan que, pese a los problemas de obsolescencia y representatividad, sigue siendo utilizada en diversas situaciones, principalmente en el ámbito español.

El estudio efectuado ofrece una perspectiva general de la clasificación, ya que aporta una solución a la problemática evidenciada. Por un lado, se ha adaptado el formato de la Nomenclatura para permitir su inclusión en cualquier aplicación de la web semántica. Por otro, se ha desarrollado un modelo que mejora su expresividad mediante la combinación de códigos Unesco y palabras clave. De esta forma se reduce la carga de decisión del usuario y se facilita la clasificación de su actividad. En este sentido, como propuesta inmediata, se plantea adaptar el modelo presentado a otros contextos, por ejemplo mediante la inclusión de los tipos "Experiencia en gestión de proyectos" o "Experiencia en colaboración empresarial".

También se ha puesto de relieve la existencia simultánea de sistemas clasificatorios de la actividad científico-tecnológica en las universidades españolas, lo que es extrapolable a otras esferas como la industrial. Recuperar la Nomenclatura Unesco como estándar permitiría unificar la actividad de clasificación en un único producto normalizador. Este trabajo constituiría un primer paso en el proceso de estandarización y universalización de esta herramienta. Así, como propuesta 
más ambiciosa, se propone el enriquecimiento de las "Áreas de coconocimiento" contenidas en la ontología a partir de las palabras clave definidas por los investigadores. Esto es, actualizar la Clasificación Uneso, con una profunda revisión de códigos y referencias cruzadas.

Obviamente, esta puesta al día de la Nomenclatura sólo puede ser llevada a cabo por expertos de las distintas áreas y su validación por organismos competentes (Méndez, 1987) y con potestad reguladora, como el CSIC o la Unesco. Estos analizarían la pertinencia de las keywords en base a parámetros aún no definidos pero entre los que podrían encontrarse el prestigio científico de quienes las utilizaron, la frecuencia de uso o el requerimiento de un grupo de expertos (por ejemplo, la Icdhs, [2012]). De esta forma, quedaría asegurado el carácter universal y normalizador de la Nomenclatura y se facilitaría el análisis de la realidad científica nacional más allá del ámbito académico. El planteamiento por tanto es evidente: normalizar la Nomenclatura y, por qué no, reactivar el programa abandonado en 1992 por la Unesco.

El modelo ontológico aprovecha la normalización de la Nomenclatura y la expresividad de las palabras clave

\section{Notas}

1. Consejería de Innovación, Ciencia y Empresa (Comunidad Autónoma de Andalucía). Orden de 3 de septiembre de 2007, por la que se regula el funcionamiento del Registro electrónico de agentes del sistemas andaluz del conocimiento para los grupos de investigación dependientes de las universidades y organismos de investigación ubicados en Andalucía y se establece su financiación.

http://deva.aac.es/include/files/investigacion/grupos/1ORDEN\%20DE\%203\%20SEPTIEMBRE\%202007.pdf

2. España. Ministerio de Economía y Hacienda. Real decreto 475/2007, de 13 de abril, por el que se aprueba la Clasificación nacional de actividades económicas 2009 (CNAE-2009). https://www.boe.es/diario_boe/txt.php?id=BOE-A-2007-8824

3. No fue posible hallar grupos de investigación en 23 de las 100 webs estudiadas, por lo que no se han incluido en la gráfica. En la mayoría de casos esto se debe a que estas instituciones son escuelas de negocios o centros adscritos que, o bien dependen de universidades ya incluidas en el estudio o no desempeñan una actividad investigadora como tal, centrando su actividad en la docencia.

4. Dirección de Investigaciones y Proyectos Académicos (DIPA), Universidad de Guayaquil. Presentación de programas y proyectos de investigación de la Universidad de Guayaquil, Convocatoria 2014.

http://www.ug.edu.ec/dipa/convocatoria_2014

5. Según versión del Ministerio de Economía y Competitividad (2011), que contiene las modificaciones del CSIC hasta 1988.

6. Stanford Center for Biomedical Informatics Research, Stanford University School of Medicine. Protègè.

http://protege.stanford.edu
7. De acuerdo con información recogida en: Secretaría de Estado de Educación, Formación Profesional y Universidades, Ministerio de Educación, Cultura y Deporte. Oferta de titulaciones. https://www.educacion.gob.es/notasdecorte/jsp/ compBdDo.do

\section{Agradecimientos}

Este trabajo ha sido posible gracias a la financiación del proyecto TSI-020302-2011-15 del Ministerio de Industria, Energía y Turismo y el fondo FEDER, y a la Universidad de Murcia a través del programa de becas predoctorales con resolución R-406/2011. Agradecemos a José Antonio Miñarro Giménez y Teddy Miranda Mena su colaboración en el desarrollo e implementación del modelo ontológico.

\section{Bibliografía}

Berners-Lee, Tim; Hendler, James; Lassila, Ora (2001). "The semantic web". Scientific American magazine, v. 284, n. 5, p. 34-43. http://dx.doi.org/10.1038/scientificamerican0501-34

Consejo Quintanarroense de Ciencia y Tecnología (2000). Clasificación Barros Sierra Unesco. Nomenclatura internacional normalizada relativa a la ciencia y la tecnología. Unesco (versión México, 2000", México.

http://coqcyt.qroo.gob. $m x / p o r t a l / p o s g r a d o /$ Clasificaci\%C3\%B3n\%20Barros\%20Sierra.pdf

Erdelen, Walter (2002). "[Respuesta a la petición de inclusión de "Diseño" como disciplina en la Nomenclatura por la Icdhs ]", 18 febrero.

http://www.ub.edu/gracmon/icdhs/docs/carta-unesco.pdf

España (1983a). “Real decreto 1804/1983, de 23 de mayo, por el que se determinan las funciones, el nivel de titulación y los procedimientos de selección para ingreso, correspondientes a las Escalas del personal científico investigador del Consejo Superior de Investigaciones Científicas". Boletín oficial del Estado (BOE), n. 157 de 02/07/1983.

http://www.boe.es/boe/dias/1983/07/02/pdfs/A18485-18486.pdf

España (1983b). "Resolución de 23 de septiembre de 1983, del Consejo Superior de Investigaciones Científicas por la que se publica la relación total de campos, disciplinas y subdisciplinas de especialización científica y tecnológica en los que desarrollan su actividad los institutos, centros e investigadores dependientes del mismo". Boletín oficial del Estado (BOE), n. 246 de 14/10/1983.

http://www.boe.es/boe/dias/1983/10/14/pdfs/A27855-27868.pdf

España (1985a) "Resolución de 20 de abril de 1985, del Consejo Superior de Investigaciones Científicas, sobre corrección de errores de la resolución de 28 de marzo de 1985, por la que se hace pública la modificación de la relación total de campos, disciplinas, subdisciplinas y especialidades científicas y tecnológicas en las que desarrollan su actividad los institutos, centros e investigadores del organismo". Boletín oficial del Estado (BOE), n. 103 de 30/04/1985.

http://www.boe.es/boe/dias/1985/04/30/pdfs/A12068-12068.pdf

España (1985b). "Resolución de 28 de marzo de 1985, del Consejo Superior de Investigaciones Científicas, por la que se hace pública la modificación de la relación total de campos, disciplinas, subdisciplinas y especialidades científicas y 
tecnológicas en las que desarrollan su actividad los institutos, centros e investigadores del organismo". Boletín oficial del Estado (BOE), n. 87 de 11/04/1985.

http://www.boe.es/boe/dias/1985/04/11/pdfs/A09581-09582.pdf

España (1986). "Resolución de 25 de marzo de 1986, del Consejo Superior de Investigaciones Científicas, por la que se hace pública la modificación de la relación total de campos, disciplinas, subdisciplinas y especialidades científicas y tecnológicas, en las que desarrollan su actividad los institutos, centros e investigadores del organismo". Boletín oficial del Estado (BOE), n. 144, de 17/06/1986.

http://www.boe.es/boe/dias/1986/06/17/pdfs/A21986-21986.pdf

España (1988). "Resolución de 10 de marzo de 1988. del Consejo Superior de Investigaciones Científicas, por la que se hace pública la modificación de la relación total de campos, disciplinas, subdisciplinas y especialidades científicas y tecnológicas en las que desarrollan su actividad los institutos, centros e investigadores del organismo". Boletín oficial del Estado (BOE), n. 180 de 28/07/1988.

http://www.boe.es/boe/dias/1988/07/28/pdfs/A23343-23344.pdf

España (2011). "Nomenclatura internacional de la Unesco para los campos de ciencia y tecnología". Subprograma de proyectos de investigación fundamental no orientada.

http://goo.gl/7WKBT2

Fernández, María-del-Rosario; Peral, Diego (2007). “Las publicaciones oficiales como medio de comunicación de la información científica y técnica a través del tiempo". Razón y palabra, v. 12, n. 56.

http://www.razonypalabra.org.mx/anteriores/n56/ fernandezperal.html

Fernández-López, Mariano; Gómez-Pérez, Asunción; Juristo, Natalia (1997). "Methontology: from ontological art towards ontological engineering". AAAl Symposium on ontologicangengineering, Stanford, pp. 33-40.

http://oa.upm.es/5484/1/METHONTOLOGY_.pdf

Icdhs. (International Conferences on Design History and Studies) (1999). "Declaración de Barcelona [por la que la asamblea de la organización acuerda pedir la inclusión de "Diseño" como disciplina en la Nomenclatura]". Ena 1a Reunión científica de historiadores y estudiosos del diseño. $V$ Primavera del diseño. Barcelona, abril.

http://www.ub.edu/gracmon/icdhs/docs/unesco.pdf

Icdhs (International conferences on design history and studies) (2012). "Unesco".

http://www.ub.edu/gracmon/icdhs/unesco.html

Martínez-Frías, Jesús; Hochberg, David (2007). "Classifying science and technology: two problems with the Unesco system". Interdisciplinary science reviews, 1 December, v. 32, n. 4, p. 315-319. http://digital.csic.es/bitstream/10261/13013/2/UNESCO_ geol_astrobio.pdf

http://dx.doi.org/10.1179/030801807X183605

Méndez, Aida (1987). "Nomenclatura internacional de la Unesco para los campos de la ciencia y tecnología. Segunda versión en español". Boletín de la Anabad, v. 37, n. 1, p. 254. http://dialnet.unirioja.es/servlet/articulo?codigo $=801853$
Ortega, José-Luis; Aguillo, Isidro F. (2007). “Interdisciplinary relationships in the Spanish academic web space: a webometric study through networks visualization". Cybermetrics. International journal of scientometrics, informetrics and bibliometrics, v. 11, n. 1.

http://cybermetrics.cindoc.csic.es/articles/v11i1p4.htm/

Pastor-Sánchez, Juan-Antonio (2012). "Nomenclatura de ciencia y tecnología de la Unesco". SKS. (Simple knowledge organization system).

http://skos.um.es/unesc6/

Pedraz-Marcos, Azucena (2005). "Enfermería no está en el Código Unesco (editorial)". Nure investigación, v. 13.

http://www.fuden.es/FICHEROS_ADMINISTRADOR/ editorial/Editorial\%2013.pdf

Suárez, Edison; Baquero, Mónica (2013). “Análisis de los proyectos del sector agropecuario financiados por Colciencias durante el año 2010". MVZ Córdoba, v. 18, n. 2, p. 359--3576. http://www.erevistas.csic.es/ficha_articulo.php?url=oai_ revista208:328\&oai_iden=oai_revista208

Torregrosa, Marta (2013). Cómo se hace una tesis doctoral. Universidad de Navarra.

http://www.unav.es/gep/Metodologia/TesisDoctoral.html

Unesco (1974). "Informe del director general sobre las actividades de la Organización en 1973”. París, Conferencia general de la Unesco $18^{a}$ reunión.

http://unesdoc.unesco.org/images/0001/000121/012174so.pdf

Unesco (1966). Problems encountered in the development of a standard international methodology of science statistics. Unesco/CS/0666.SS-80/5. Paris: Oficina Estadística. http://unesdoc.unesco.org/images/0014/001437/143792eb.pdf http://unesdoc.unesco.org/images/0001/000121/012174so.pdf

Unesco (1988). Proyecto de Nomenclatura internacional normalizada relativa a la ciencia y la tecnología. Revisión 1. Unesco/ND/ROU/257. París: Unesco.

http://unesdoc.unesco.org/images/0008/000829/082946sb.pdf

Urdín-Camino, Carmen (2001). "La edición de revistas científicas en España”. En: Giménez-Toledo, Elea; Gómez-Caridad, Isabel; Martín-Sempere, María-José; Páez-Mañá, Jorge; Román-Román, Adelaida; Vázquez-Valero, Manuela. La edición de revistas científicas: Guía de buenos usos. Centro de Información y Documentación Científica. ISBN: 8400079167 http://digital.csic.es/handle/10261/4347

Urdín-Camino, Carmen; Alcaín, María-Dolores (2004). "Bases de datos CSIC en la Red". Hipertext.net, v. 2.

http://elis.da.ulcc.ac.uk/9009/1/Bases_de_datos_CSIC_en_ la_red.pdf

Urdín-Camino, Carmen; Morillo, Fernanda (2000). "Producción científica de Andalucía en las bases de datos Science citation index e índice español de ciencia y tecnología". Revista española de documentación científica, v. 23 n. 4, p. 379-394. http://redc.revistas.csic.es/index.php/redc/article/viewArticle/327

W3C (2012). OWL 2 web ontology language. Documenw overview second edition).

http://www.w3.org/TR/ow/2-overview 